\title{
Protée
}

\section{Une posture éthique en deçà des valeurs ?}

\section{Louis Panier}

Volume 36, numéro 2, automne 2008

Éthique et sémiotique du sujet

URI : https://id.erudit.org/iderudit/019021ar

DOI : https://doi.org/10.7202/019021ar

Aller au sommaire du numéro

Éditeur(s)

Département des arts et lettres - Université du Québec à Chicoutimi

ISSN

0300-3523 (imprimé)

1708-2307 (numérique)

Découvrir la revue

Citer cet article

Panier, L. (2008). Une posture éthique en deçà des valeurs ? Protée, 36(2), 69-78. https://doi.org/10.7202/019021ar

\section{Résumé de l'article}

L'éthique et la sémiotique ont en commun de poser la question de l'identité et de la construction de valeurs et de décrire les modalités de constitution d'un sujet relatif aux valeurs et à leur mise en pratique. Après avoir repris la problématique sémiotique classique des valeurs et des objets-valeurs, cet article envisage, à la suite de l'analyse d'un court récit-parabole, la possibilité d'une constitution éthique du sujet instauré en deçà des valeurs et des objets où elles s'investissent, dans la posture qu'il prend relativement à l'altérité et à la nécessaire absence d'une instance d'énonciation. 


\section{UNE POSTURE ÉTHIQUE EN DEÇÀ DES VALEURS?}

LOUIS PANIER

L'éthique et la sémiotique ont en commun de poser la question de l'identité et de la construction des valeurs et du lien qu'entretient avec les valeurs un sujet engagé dans une pratique, quel qu'en soit le domaine d'application: économique, politique, médical, juridique...

VALEURS, SUJETS, PRATIQUES

L'articulation d'un sujet aux valeurs et aux pratiques fait partie des constructions fondamentales de la sémiotique. Les éléments de la grammaire prévoient en effet cette double orientation de l'actant-sujet, d'une part en direction des valeurs investies dans des objets, d'autre part en direction d'une pratique orientée, en termes de programme, vers la valeur qui la finalise. La question éthique peut se poser du fait de cette double orientation de l'actant-sujet. Elle concerne en effet l'action, et pas seulement la jonction d'un sujet à un objetvaleur; il s'agit du sens de l'action et des valeurs investies et impliquées dans l'action elle-même. On pourra distinguer un double investissement des valeurs, dans l'actant-objet corrélatif de l'actant-sujet, d'une part, dans le faire corrélatif au sujet opérateur modalisé, d'autre part. Si le premier cas concerne plutôt le sujet «idéologique», le second intéresse plus directement le sujet «éthique»; il appartient donc à une approche sémiotique de bien distinguer ces deux formes d'investissement, d'en mesurer la différence et les effets sur la constitution du sujet.

La première définition de la valeur concerne l'investissement dans des objets syntaxiques, et s'établit dans le cadre d'une axiologie. L'articulation paradigmatique des valeurs (virtuelles) donne lieu à des «taxinomies valorisées que l'on peut désigner du nom d'axiologies" (Greimas et Courtés, 1979: 179).

Lorganisation syntagmatique des valeurs les convertit dans «des modèles qui apparaissent comme des potentialités de procès sémiotiques [...], on peut les considérer comme des idéologies (au sens restreint, sémiotique, de ce mot)»(ibid.). L'idéologie dynamise les structures axiologiques sous forme de procès et y engage des sujets. Axiologie et idéologie déterminent donc le champ des valeurs où un 
sujet trouve à se définir. Le champ des valeurs précède l'instauration du sujet et Greimas a pu analyser en termes de construction modale les dispositifs d'investissement de la valeur dans l'objet syntaxique (1983: 93-102).

La seconde perspective concerne le sens et la valeur de l'action; non plus les objets comme lieux d'investissement des valeurs, mais le parcours même, son orientation et sa fonction dans la constitution d'un sujet sémiotique. L'éthique ici porte sur le cours d'action par lequel un sujet peut se définir. Ici encore, on retrouve des préoccupations "classiques» de la sémiotique narrative qui, avec les énoncés caractéristiques de la manipulation et de la sanction, a pu se donner une représentation syntaxique de la problématisation du sens de l'action projetée ou réalisée. Classiquement, la sanction évalue la conformité de l'action à un système de valeurs préalablement posé (dans la phase de manipulation), ce ne sont pas l'action comme telle ou le parcours du sujet qui sont pris en charge ${ }^{1}$. De ce fait, on peut se demander si la prise en considération du parcours luimême et des rapports qu'il entretient avec le sujet qu'il instaure et manifeste en tant que sujet éthique ne vient pas remettre en question la seule conformité aux valeurs pour la définition de la position éthique. Y a-til, en deçà (ou au-delà) de la position des valeurs, une place pour la convocation et l'instauration d'un sujet?

Nous illustrerons cette question en analysant rapidement une parabole évangélique (Panier, 2003a; 2003b), qui nous semble poser assez clairement la question des valeurs, des formes d'investissements dans des objets ou dans des parcours d'action, et finalement articuler la question éthique à la question de la constitution du sujet, et cela en deçà des systèmes de valeurs.

Intéressé par la pratique de la lecture sémiotique, nous nous demanderons pour finir ce qu'il en est d'une éthique de la sémiotique et du type de sujet qu'une telle pratique appelle. Là encore, on pourra observer comment la lecture sémiotique - prise comme acte énonciatif - convoque un sujet en deçà des «valeurs» de sens.
CROIRE DANS LA PLUS PETITE CHOSE: un récit-parabole Le récit-parabole, extrait du chapitre 19 de l'Évangile de Luc ${ }^{2}$, est le suivant:

(12) [...] Un homme de haute naissance se rendit dans un pays lointain pour recevoir la dignité royale et revenir ensuite.

(13) Appelant dix de ses serviteurs, il leur remit dix mines et leur dit: "Faites des affaires jusqu'à ce que je vienne».

(14) Mais ses concitoyens le haïssaient et ils envoyèrent à sa suite une ambassade chargée de dire: "Nous ne voulons pas que celui-ci règne sur nous".

(15) Et il advint qu'une fois de retour, après avoir reçu la dignité royale, il fit appeler ces serviteurs auxquels il avait remis l'argent, pour savoir ce que chacun avait fait produire.

(16) Le premier se présenta et dit: «Seigneur, ta mine a rapporté dix mines».

(17) «C'est bien, bon serviteur, lui dit-il; puisque tu t'es montré fidèle en une toute petite chose ${ }^{3}$, reçois le pouvoir sur dix villes».

(18) Le second vint et dit: «Ta mine, Seigneur, a produit cinq mines".

(19) À celui-là encore il dit: "Toi aussi, sois à la tête de cinq villes".

(20) L'autre vint aussi et dit: «Seigneur, voici ta mine que je gardais déposée dans un linge.

(21) Car j'avais peur de toi, qui es un homme sévère, qui prends ce que tu n'as pas mis en dépôt et moissonnes ce que tu n'as pas semé».

(22) «Je te juge, lui dit-il, sur tes propres paroles, mauvais serviteur. Tu savais que je suis un homme sévère, prenant ce que je n'ai pas mis en dépôt et moissonnant ce que je n'ai pas semé.

(23) Pourquoi donc n'as-tu pas confié mon argent à la banque? À mon retour, je l'aurais retiré avec un intérêt».

(24) Et il dit à ceux qui se tenaient là: «Enlevez-lui sa mine, et donnez-la à celui qui a les dix mines».

(25) «Seigneur, lui dirent-ils, il a dix mines!»

(26) «Je vous le dis: à tout homme qui a l'on donnera; mais à celui qui n'a pas on enlèvera même ce qu'il a».

(27) Quant à mes ennemis, ceux qui n'ont pas voulu que je règne sur eux, amenez-les ici et égorgez-les en ma présence.

Nous nous intéresserons particulièrement à l'objet figuré par la «mine», aux différents dispositifs d'investissement de valeur dont il est le lieu et aux différentes relations constitutives de sujets qui s'y 
nouent. Nous examinerons également comment ce récit met en rapport des parcours de sujet et leur définition en termes de valeur. Entre les valeurs d'objet et les valeurs d'action, nous suggérons de lire, dans ce récit, une certaine problématisation de l'éthique, dont une lecture sémiotique doit pouvoir décrire la cohérence.

Si la mine est un objet, elle figure sous plusieurs modes. Il y a la mine donnée à chaque serviteur, il y a les mines produites, mentionnées par les deux serviteurs et sur lesquelles s'opère la sanction du roi (dix pour l'un, cinq pour l'autre), il y a enfin la mine enlevée au troisième serviteur et attribuée au premier, et qui déclenche la surprise de l'entourage royal et l'explication du roi ${ }^{4}$.

Mise en place d'un cours d'action

La structure narrative est assez simple. Le don des mines et le départ du maitre correspondent à une phase de manipulation où se trouve engagé un programme pour les serviteurs et la figure de cet objet, la mine, se trouve mise en rapport avec des sujets, mais avec une valeur qui reste non précisée. Le programme reste assez indéterminé en termes de valeur (faire «des affaires " ${ }^{5}$ ). La mine, selon l'encyclopédie, représente une certaine somme d'argent ${ }^{6}$, mais elle reste ici une valeur potentielle soumise à la seule activité des sujets. S'il faut faire (des affaires) avec cette mine, la valeur de celle-ci n'est autre que la valeur de l'action qu'elle occasionne.

Au retour du roi, on reconnaît la sanction qui comporte, de façon très canonique, une information sur la performance, une évaluation et la rétribution par le Destinateur épistémique. Cette dernière n'est pas effectuée dans le récit, il n'y a aucun transfert d'objet pratique: les serviteurs ne rendent pas les mines au roi, on ne raconte ni l'attribution des villes, ni la soustraction de la dernière mine au troisième serviteur et son attribution au premier. Il n'y a que du discours: une déclaration et un débat sur les valeurs, que vient conclure la mystérieuse réponse du roi: «À tout homme qui a l'on donnera; mais à celui qui n'a pas on enlèvera même ce qu'il a».
Les annonces de rétributions manifestent les valeurs investies dans les objets en relation avec l'engagement ou le non-engagement des sujets dans le cours d'action proposé. Ces rétributions obéissent à deux formes: attribution $d u$ pouvoir sur les villes pour les deux premiers serviteurs, retrait de la mine au troisième et don au premier. Elles manifestent ce qu'il en est du statut des sujets en fonction des rapports aux valeurs investies dans les objets d'une part, au parcours d'action d'autre part: nous retrouvons bien la double direction de la question éthique.

Deux types (ou deux cas) de sujet sont posés: pour les deux premiers serviteurs, la production réussit; pour le troisième, il n'y a pas à proprement parler échec car la performance n'a pas été engagée, c'est donc bien du côté de l'instauration des sujets, des conditions de leur engagement dans un parcours, et non de leur compétence (pouvoir-faire ou savoir-faire) que se pose le problème et qu'il faudra observer la relation aux valeurs.

\section{Deux serviteurs ...}

Les deux premiers serviteurs informent de la performance accomplie ( «ta mine a rapporté dix mines [...] a produit cinq mines»), mettant en avant la mine elle-même et non leur propre compétence. $\mathrm{La}$ réponse du roi attribue la réussite du parcours au statut fiduciaire du sujet («bon serviteur», «foi » 7 ), au contrat fiduciaire supposé lors du départ du maître et manifesté par le don de la première mine. La valeur économique est subordonnée à la position énonciative, elle-même référée à la non-valeur (la plus petite chose). L'énoncé de la sanction manifeste une structure contrastée, voire paradoxale, des valeurs: grande quantité produite versus foi «dans la plus petite chose». La position initiale du sujet, qui n'est dévoilée, par le roi, qu'à la fin du parcours, concerne bien le rapport, l'engagement du sujet à un dispositif fiduciaire qui suppose que l'objet attribué soit à la fois décisif et sans-valeur ${ }^{8}$.

On peut donc s'interroger sur le statut sémiotique de cet «objet-sans-valeur» et sur sa fonction dans l'instauration des sujets. Au terme du parcours de 
production seulement, les mines peuvent être converties en "valeur» (mines = villes) par une opération d'équivalence de type arithmétique entre les mines produites et les villes sur lesquelles les serviteurs auront le pouvoir (dix pour dix, cinq pour cinq). On peut parler de transposition, de transfert d'isotopie ou d'opération métaphorique: le pouvoir sur les villes signifie (manifeste) la valeur de la production des mines. Le sens ou la valeur du parcours d'action des serviteurs se trouvent ainsi pris en charge par deux dispositifs sémiotiques différents: un processus métonymique lorsque les mines se multiplient et s'enchaînent dans un système de valeurs purement cumulatif et comptable (dix pour un; cinq pour un), processus relevant d'une rationalité pratique; un processus métaphorique lorsqu'une transposition d'isotopie (un saut) interprète le premier processus au moment même où il l'interrompt, processus relevant d'une rationalité sémiologique si le pouvoir acquis est le signifiant de la valeur de la production réalisée: le pouvoir sur les villes vient identifier des sujets qui n’assumaient pas le parcours des mines («ta mine a rapporté...») $)^{9}$.

... et un troisième

Le récit développe plus largement la sanction du troisième serviteur dans un dispositif énonciatif assez complexe. Le serviteur restitue la mine qui lui a été confiée et justifie son non-engagement dans le programme de production par des considérations subjectives et pathémiques concernant et lui-même ( $i$ 'avais peur») et son maitre ( tu es un homme sévère»).

La restitution de la mine («voici ta mine») présuppose pour cet objet un statut d'objet-valeur installé dans un dispositif réciproque d'échange (disjonction/conjonction) entre deux sujets. S'il y a ici un contrat avec le maître, il porte sur la valeur investie dans cet objet et sur une anticipation de son devenir: la mine doit entrer dans un processus de production et le roi est censé s'emparer des résultats de cette production ${ }^{10}$, «prenant ce [qu'on n'a] pas mis en dépôt», «moissonnant ce [qu'on n'a] pas semé». Il s'agit de la production comme «valeur» objectivable, appropriable, selon une rationalité pratique, opposée à la rationalité sémiologique mise en œuvre dans la conversion mines/villes. La position imaginée du maître (sévère) est exactement l'inverse de la position réelle du roi vis-à-vis des serviteurs: il ne prend pas les mines produites, il les interprète, il leur donne sens, il en produit des signes. La sanction du roi vérifie le procès de production lui-même et porte intérêt à l'objet initial, non pas pour son statut d'objet-valeur, mais pour son statut fiduciaire (foi «dans la plus petite chose").

Ce récit ne repose pas simplement sur l'opposition entre différents systèmes de valeurs investis dans des structures narratives. Il met en discours différents dispositifs éthiques, c'est-à-dire différents modes d'instauration de sujets, à partir des modes de relations aux valeurs, aux objets et aux parcours d'actions. Il le fait en développant le parcours d'une figure d'objet: la «mine», d'abord posée figurativement comme prototype de la "valeur» ${ }^{11}$, se trouve ensuite déployée entre les sujets, comme «à distance» des valeurs qui pourraient s'y trouver investies.

La sanction du troisième serviteur pose un problème à l'analyse: en quoi la soustraction de la mine fait-elle système avec le mode de rétribution des deux premiers serviteurs? La mine initialement confiée par le maître, et que le serviteur voulait restituer, est finalement prise par le roi pour être donnée au premier serviteur (celui qui a produit dix mines). Mais elle ne fait pas nombre pour s'ajouter aux mines produites (métonymie), elle ne fait pas l'objet d'une conversion du type mine $=$ ville (métaphore): elle demeure comme un objet non investi par une valeur, un objetsans-valeur, qui fait rappel du statut réel de la mine initialement confiée. Passant du troisième au premier serviteur, cette mine est excessive et elle marque la limite d'un système établi sur les valeurs; et c'est cela qui fait problème ( "Seigneur, lui dirent-ils, il a dix mines!») et c'est cela qui est articulé à la loi énoncée par le roi ( «Je vous le dis: à tout homme qui a l'on donnera; mais à celui qui n'a pas on enlèvera même ce qu'il a»). 
La marque du sujet

Le récit nous conduit ainsi vers un dispositif caractérisé par la non-valeur et le non-signe: cette dernière mine reste un objet non investi de "valeur" pour un sujet, un objet non interprété en termes de valeur, une «figure» qui, absente ou présente, est la marque d'un sujet désigné ("dé-signé») à la parole d'un autre. Nous proposons de parler alors d'une rationalité figurale qui fait écart à la rationalité pratique et à la rationalité sémiologique que le récit met également en place.

Au serviteur à qui elle est prise, cette mine fera défaut, non pas à la manière d'un manque d'objetvaleur qu'une performance pourrait liquider, mais à la manière d'une marque qui l'ordonne à cet objet sans valeur. N'appartenant à aucun des dispositifs métonymique ou métaphorique d'investissement de valeur mis en place précédemment, elle est également pour le premier serviteur une marque plutôt qu'un signe. Marque identifiante, cet objet-sans-valeur fait rappel de «la plus petite chose» à propos de laquelle se sont ancrées la fidélité ou la peur. Alors que la conversion mines $=$ villes vient signifier (décoder) le point terminal du processus réalisé, cette ultime mine vient faire rappel (retour) du point initial du processus productif. Elle n'est pas un objet-valeur, elle n'est pas un signe, elle est une marque, une grandeur «figurale», la trace de l'acte d'énonciation dans lequel un sujet s'est trouvé suscité en face d'une altérité qui s'absente. C'est à partir de cette "petite chose" posée et re-posée que s'instaure pour un sujet la question (à laquelle un autre répond) de ce qui excède (par défaut, pourraiton dire) le sens et les valeurs. Résumons-nous.

- Prise dans une rationalité pratique, la «mine», comme signe-renvoi, permet de déployer une configuration financière, comme le montre le dialogue du roi avec le dernier serviteur.

- Dans la phase médiane du récit, la mine comme figure est prise dans une opération métonymique: les mines se succèdent et se multiplient (tantôt dix, tantôt cinq pour un), elles n'ont de valeur quantitative qu'à être multipliées.

- Au retour du roi, la figure est reprise dans un dispositif métaphorique, opération interprétative qui installe la mine dans un dispositif de signe, articulant l'isotopie financière et l'isotopie politique.

- À la fin du parcours, la figure assure, par son rappel, par sa présence surnuméraire pour l'un et son retrait pour l'autre, la fonction de marque. Loin d'être un signe interprétable ou convertissable en valeur (comme les mines associées à des villes), elle a le statut d'«in-signe», de non-signe désignant la singularité d'un sujet dans l'acte énonciatif qui l'instaure et pour le parcours figuratif qui le représente.

Une approche sémiotique de la question éthique peut proposer des modèles de la construction et de l'articulation des valeurs pour un sujet, des formes d'assomption, des cours d'actions et des pratiques par un sujet. Le récit que nous avons lu nous semble pouvoir être versé à ce dossier; sans doute parce que sa manière d'articuler le sujet, les valeurs et les pratiques est un peu paradoxale ${ }^{12}$. C'est bien la notion de "valeur» qui est en cause dans ce récit: en tant que somme d'argent ${ }^{13}$, la mine est typiquement une figure d'objet-valeur et la consigne donnée par le maître installerait typiquement un programme conforme à ces valeurs - l'adhésion à ce programme serait ce qui sépare les bons serviteurs et l'autre serviteur et ce qui est sanctionné par un partage du pouvoir...

L'analyse que nous avons pu faire de ce court récit permet d'y déceler, au-delà du cadre "évangélique», des dispositifs sémiotiques un peu différents et d'en extraire des modèles de constitution du sujet qui concernent bien les questions éthiques: la question du rapport d'un sujet à la valeur, celle de la valeur des valeurs et celle du point d'émergence des valeurs pour un sujet. Sur ces questions, cette parabole propose des modèles qui peuvent sans doute être développés.

\section{UN MODĖLE POUR UN DISPOSITIF ÉTHIQUE?}

L'éthique pose la question du sens et de la valeur de l'action, elle suppose un sujet engagé dans une 
pratique, dans un cours d'action. Sens et valeur excèdent en quelque sorte les valeurs elles-mêmes, d'où l'importance, dans le schéma narratif canonique, des phases de manipulation et de sanction qui mettent en place un tiers-actant (Destinateur), des procédures d'évaluation et une catégorie d'objetsmessages manifestant justement la valeur des valeurs et l'isotopie de leur qualification.

Tel serait le regard sémiotique sur l'éthique dans la perspective classique du parcours génératif et de l'ancrage objectal des valeurs. Il permet de formaliser des dispositifs éthiques, de modéliser des choix mis en œuvre dans différentes constructions discursives ou pratiques. Il convient toutefois de complexifier le modèle sur plusieurs points et de l'interroger. Notre récit s'intéresse bien aux bornes du parcours narratif, mais il ne fait pas reposer l'instauration et la reconnaissance de sujets sur l'adhésion à un système de valeurs préalablement articulées et sur leur déploiement dans un parcours narratif. Il y a ici de l'insu dans la constitution des sujets, et une forme de débrayage qui atteint la relation d'investissement des valeurs dans les objets, qui dissocie la liaison sujetvaleurs-objet et pose dans l'écart ainsi dessiné la place d'un sujet défini dans un espace d'énonciation et représenté par un "objet sans valeur", grandeur figurale, qui l'identifie à la manière d'une marque.

Si l'éthique surplombe l'univers des valeurs (qui peuvent être investies dans les objets syntaxiques) engagées dans des pratiques, elle pose d'une part la question des rapports entre cet investissement et l'instauration d'un sujet et, d'autre part, la question de la signification (et de la compréhension) du parcours même du sujet. La question éthique devient la question du statut du sujet par rapport à l'univers de valeurs (idéologique) et par rapport au parcours en quoi se déploie une pratique. La question éthique se pose dans l'écart que suppose, dans l'un et l'autre cas, ce rapport.

Cette question nous a paru assez bien posée dans la forme même du récit-parabole, où nous avons observé la tension pour un sujet, entre l'investissement des valeurs dans les objets et l'engagement dans une pratique, dans un cours d'action dont le sens et la valeur restent déterminés par un choix qui relève de la fiducie plus que de l'adhésion aux valeurs ou à l'investissement des objets. L'éthique concerne alors le statut et l'identité du sujet représenté par une figure, un "signifiant" pris dans un parcours, dans un enchaînement d'actions (métonymique), signifié dans des opérations métaphoriques de conversion idéologiques (faisant retour au système des valeurs), mais finalement rappelé, revenant comme marque-du-sujet, attestant l'acte par lequel le sujet a été posé en relation à une altérité qui s'absente. Le statut éthique du sujet suppose ici la considération paradoxale d'un «objetvaleur-sans-valeur» (comme la mine dans notre parabole) qui n'atteste l'instauration d'un sujet qu'à être justement neutralisé comme objet-valeur. Dans notre récit, la considération de la mine comme objet investi d'une valeur, comme objet-valeur de transfert susceptible d'être attribué, repris, conservé, bloque pour le troisième serviteur toute entrée dans la pratique et dans le cours d'action. Il conviendrait de revenir sur le statut sémiotique de cet «objet-valeursans-valeur». Sa nécessité oblige peut-être à reprendre la question de l'investissement de la valeur dans les objets et à suggérer que l'instauration du sujet de l'action suppose une forme particulière de débrayage qui fait division dans l'objet-valeur et dans le sujet qui s'en trouve déterminé.

Au niveau élémentaire de la syntaxe narrative, l'objet n'a d'autre réalité que d'être un actant défini par la fonction qui le relie à l'actant sujet. L'objet semble un lieu qu'une valeur vient remplir, ou «investir» ${ }^{14}$. Cette notion d'investissement, utilisée par Greimas, mérite qu'on s'y arrête. Dans le Dictionnaire raisonné de la théorie du langage, Greimas et Courtés la définissent dans la logique du parcours génératif de la signification comme «une procédure par laquelle une structure syntaxique donnée se voit attribuer des valeurs sémantiques préalablement définies» (1979: 196). On peut ainsi parler «d'une composante sémantique autonome, relativement indépendante de la composante syntaxique» (ibid.). La 
notion d'investissement est à nouveau utilisée pour rendre compte des états existentiels des valeurs (virtuelles, actualisées, réalisées). Virtuelles dans les structures du carré sémiotique, les valeurs sont actualisées quand elles peuvent recevoir "l'investissement complémentaire de la catégorie thymique» (ibid.: 414), cet investissement n'étant concevable que dans la mesure où la valeur est mise en relation avec un sujet (dans les cadres prévus par les structures narratives de surface). Investie dans les actants-objets des énoncés d'état, et lorsque la conjonction avec l'objet-valeur s'effectue au profit du sujet d'état, la valeur actuelle se trouve transformée en valeur réalisée (ibid.: 415). Il y a valeur pour un sujet moyennant l'investissement dans un objet et l'élaboration thymique et modale de la valeur ${ }^{15}$.

Le parcours narratif dans lequel un sujet est installé ne peut s'inaugurer que par la mise en perspective d'un objet investi d'une valeur et modalement joint à un sujet. Tout se passe comme si la fonction du Destinateur consistait à proposer, comme structure véridictoire, l'investissement sémantique et modal de l'objet et à opérer la transformation modale qui lie cet objet comme objetvaleur pour un sujet, la relation $S \rightarrow$ Ov étant caractéristique de l'axe du désir dans la terminologie traditionnelle du modèle actantiel.

On peut alors reconnaittre pour cet objet-valeur une structure sémiotique constituée de deux plans: celui de valeurs articulées dans les dispositifs catégoriels et modaux et celui des figures, organisé par les dispositifs narratifs et discursifs et sur lequel les objets peuvent être caractérisés par leur "présence» 16 . Sans doute le sujet «idéologique» est-il lié au monde des valeurs. La valeur s'investit dans un objet qui peut en être le lieu, le support, voire le signifiant dans un cadre idéologique donné (Panier, 1980); il faut toutefois compter avec les objets en tant que "grandeurs figuratives", renvoyant au monde naturel et sensible, mais également aux expériences intersubjectives et énonciatives dont ils sont la trace (Martin, 1995: 143). Notre récit-parabole pointe vers ce lieu de la question éthique, vers ce point d'écart qui dissocie la structure idéologique du signe (où l'objet serait le signifiant de la valeur) et qui isole aussi l'objet de sa puissance pathémique (seul le troisième serviteur est un sujet passionnel: «j'avais peur»), pour ne conserver que «la plus petite chose», la simple marque pour un sujet suscité par une altérité qui s'absente, et déployé en parcours entre deux actes d'énonciation.

\section{UNE ÉTHIQUE DE LA (PRATIQUE) SÉMIOTIQUE?}

Ce récit-parabole, tel que nous l'avons abordé, nous semble fournir une structure pour la posture éthique du sujet, une certaine «forme de vie». Cette structure est susceptible de s'appliquer également à la pratique de la sémiotique, c'est-à-dire à l'expérience d'un sujet engagé dans la lecture, dans la construction d'une cohérence de discours sur les objets qui se donnent à percevoir (Panier, 1998). Il peut être question de posture éthique d'un sujet si l'on s'interroge sur les rapports qu'un sujet (interprète ou lecteur) entretient avec les valeurs qui peuvent être associées à ces objets en fonction des diverses saisies qui peuvent être les siennes ${ }^{17}$.

La posture de l'interprète peut être investie d'une compétence que nous dirons «encyclopédique», qui déploie la signification des objets dans la reconnaissance et dans la construction de réseaux conformes à la sémiotique du monde naturel. Renvoyer ainsi l'objet à sa place dans la sémiotique du monde naturel, c'est négliger sa capacité à entrer «en discours" dans des ensembles signifiants relevant d'une instance énonciative. Renvoyer chaque objet à sa position dans les réseaux du monde naturel, c'est en quelque sorte rester au seuil du parcours de l'interprétation. Le sujet se détermine alors par le savoir (et le pouvoir-savoir). La lecture est une "quête" mobilisée par le savoir (manquant ou acquis) et tendue vers cet objet-valeur qu'est alors le savoir.

La posture de l'interprète peut également être investie d'une compétence qui s'adapte principalement au caractère thématique du dispositif discursif figuratif et vise à associer figuratif et thématique dans des constructions de type 
symbolique. Le discours convoque alors un sujet relatif aux valeurs (et aux systèmes de valeurs) axiologiques et idéologiques, un sujet susceptible d'intégrer et d'assumer les univers de valeurs à l'oeuvre dans le discours ${ }^{18}$. Derrière le jeu des figures, c'est l'armature thématique qui charpente un sujet apte à intégrer (à croire) les formes de vie manifestées par le discours et à inscrire son vouloir dans le champ axiologique déployé par le texte: le texte manifeste, pour le sujet du discours, la cohérence d'un système de valeurs investies dans des dispositifs narratifs et figuratifs (le «monde du texte»). On pourra alors parler de sujet idéologique, en reprenant la définition de l'idéologie donnée par Greimas.

On pourra caractériser une troisième posture $\mathrm{du}$ sujet interprète ou lecteur sémioticien. On parlera d'une posture langagière (ou littérale) pour autant qu'elle fait appel à la langue (non pas au sens saussurien du terme, ni au sens de langue naturelle, mais selon la définition de Lacan, soit «lalangue» qui porte toujours trace de l'énonciation préalable - ou parole - qu'elle manifeste et recèle dans la lettre). Cette posture s'adapte principalement au caractère discursif de l'enchaînement figuratif ${ }^{19}$ et à l'écart maintenu entre les parcours de figures et les valeurs (le sens) qui peuvent leur être associées. Ce qui caractérise les figures en discours, c'est justement qu'elles sont... en discours, qu'elles tiennent leur existence et leur efficace de leur installation dans un enchaînement qui atteste un acte énonciatif, instance que ne peuvent réduire ou périmer ni la résonance référentielle, ni la résonance thématique du texte, instance qui n'a d'autre «représentation» que le parcours qui lui répond. Le "déroulement de la parole figurative» (Greimas, 1993: 383) dans la parabole est le prototype d'un discours où la figure "particularise et signe l'originalité du langage pris dans l'énonciation", selon une expression de J. Calloud, c'est-à-dire du langage en tant que s'y joue la possibilité d'être pour un sujet. Paradoxalement, cette possibilité s'atteste et se joue dans la figure (et en figures), dans ce supplément de sens signalé par les rhétoriciens, dans cet excès par rapport aux valeurs, supplément de valeur qui peut être aussi bien défaut de sens, trouble où se manifeste la passion du sujet, ou le sujet de la passion ${ }^{20}$ qui ne peut être confondu avec celui qui est dépositaire (ou en quête) du savoir, ni avec celui qui s'ordonne aux formes des systèmes axiologiques.

Les préconditions du sens pour un sujet seraient à chercher dans ce qui le lie à la langue en tant que discours: traversée d'énonciation, elle est le dépôt de la parole. Si la langue est le dépôt de la parole parce qu'elle est d'abord reçue (et s'indique de cette manière), il conviendrait de faire place en sémiotique, pour définir les conditions ou préconditions du sens et la posture d'un sujet, à ce lien de la langue, de la parole et du corps, et à «la toute petite chose» qui en marque le nouage et dont notre récit-parabole signale qu'elle est le départ qui lance le cours d'action et que celui-ci révèle.

C'est donc entre les figures mises en discours, dans l'écart figural qui les vide et les articule tout à la fois (pression de la langue ou du discours sur le sens constitué, tension entre l'axe métonymique du cours d'action et l'axe métaphorique de son interprétation) qu'il conviendrait de chercher cet inestimable objet à quoi peut tenir la constitution éthique d'un sujet de la lecture, d'un sémioticien interprète. Et l'on pourrait alors gloser la parole du roi dans la parabole: à celui qui a cet objet vacillant entre les figures mises en discours, il sera donné et il aura du surplus (un statut, une identité posée au-delà ou en deçà des valeurs); à celui qui n'a pas cela, même ce qu'il a (le sens thématisé, les valeurs axiologisées, les représentations stabilisées, les signes constitués) lui sera enlevé.

Une éthique de la sémiotique serait alors tendue vers ce qui, au-delà du sens (qui pourtant fait l'objet principal de la quête), constitue une place de sujet articulé à l'écart et au nouage entre des grandeurs figuratives et les structures thématiques susceptibles de les investir comme valeurs. 


\section{N O TES}

1. On peut rappeler les critiques de Ricœur à l'égard de la sémiotique narrative et reprendre, pour les examiner, les notions d'intrigue et de configuration, censées saisir l'action comme sens (Panier, 2008).

2. Traduction de la Bible de Jérusalem.

3. Littéralement: «croyant dans la plus petite chose».

4. Ce récit articule deux parcours narratifs, celui de l'homme noble qui va recevoir la royauté et revient, et celui des serviteurs qui sont invités à «faire des affaires» avec les mines. Ces deux parcours sont syntagmatiquement liés: l'opération demandée aux serviteurs est délimitée par le parcours royal (départ/retour) et la royauté acquise semble être la compétence requise pour la sanction finale des serviteurs et des concitoyens. Discursivement, la corrélation des deux parcours organise dans le texte une articulation spatio-temporelle spécifique où l'on peut distinguer: ici versus ailleurs (spatialisation), temps de l'absence versus temps de la présence (temporalisation indexée sur un acteur et non sur des repères chronologiques). La production des mines par les serviteurs est déterminée par rapport à une temporalité hétéronome: initiée par le maître en son départ, elle est interrompue par la venue du roi et non pas achevée par l'obtention d'un résultat programmé.

5. Dans le texte grec, le verbe pragmateuomai (s'efforcer, se donner de la peine) ne définit pas d'emblée une activité économique ou commerciale.

6. Une mine correspond, dit-on, au salaire de cent journées de travail.

7. Luc 19, 17: "Tu as été croyant dans la plus petite chose".

8. Même si une mine correspond, dit-on, au salaire de cent journées

de travail, elle est considérée ici en dehors de cette «valeur». À la

différence du troisième serviteur pour lequel la mine donnée est un objet de valeur redoutable!

9. C'est le retour du roi qui interrompt et vient donner sens à la multiplication des mines qui, par elle-même, serait un processus indéfini dont, d'ailleurs, aucun sujet ne peut se recommander ("ta mine a produit x mines "). On n'ose pas imaginer ce qui serait advenu de ce processus de production si le roi n'était pas revenu! Le parcours d'action prend sens d'être interrompu.

10. Et à juste titre semble-t-il: «tu savais que je suis un homme sévère, prenant $[. .$.$] »). Le roi valide l'opinion que le serviteur a de lui : quel est$ alors son statut éthique dans le cours du récit?

11. À la manière du trésor dans les récits traditionnels: le stéréotype de l'objet-valeur est justement celui sur lequel l'énonciation narrative opère les transformations les plus radicales.

12. Ce n'est pas ici le lieu de développer l'éventualité d'une éthique "évangélique "; il s'agit seulement de souligner une forme particulière de la question éthique, susceptible d'enrichir la problématique sémiotique.

13. «Cent deniers, ou le salaire de cent journées de travail» (Évangile selon Luc, trad. Sœur Jeanne d'Arc, Les Belles Lettres, Desclée de Brouwer, 1986, 153)
14. «En tant qu'actants, les objets syntaxiques sont à considérer comme des positions actantielles, susceptibles de recevoir des investissements soit de projets des sujets (on parlera alors des objets de faire) soit de leurs déterminations (objets d'état). [...] L'objet - ou objet de valeur se définit alors comme le lieu d'investissement des valeurs (ou des déterminations) avec lesquelles le sujet est conjoint ou disjoint " (Greimas et Courtés, 1979: 259).

15. «On voit, à la suite de ce réexamen, que ce que nous avons pris l'habitude d'appeler valeur en partant d'objets de valeur est en réalité une structure modale: $\mathrm{V}=$ me $(\mathrm{s})$ où " $\mathrm{s}$ " désigne une grandeur sémique quelconque, sélectionnée lors de la conversion, et "me" une structure modale dont le premier terme " $\mathrm{m}$ " désigne une des modalités sélectionnées, et " $\mathrm{e}$ ", la relation existentielle modifiée par la modalisation. [...] Remarque: Il conviendrait peut-être de distinguer, dans l'écriture, un "v" minuscule, servant de symbole à la valeur axiologique, d'un $\mathrm{V}$ majuscule, désignant la valeur déjà modalisée. [...] Une telle valeur modalisée, une fois inscrite dans l'objet, lui-même actant de l'énoncé d'état, se trouve alors soumise aux opérations de jonction (conjonction et disjonction) effectuées par le sujet de faire (situé en syncrétisme à l'intérieur d'un même acteur, ou représenté par un acteur autonome et distinct)» (Greimas, 1983: 100).

16. Voir C. Zilberberg (2006) et E. Landowski (2004).

17. Nous prenons ici le terme de "saisie" au sens que lui donne J. Geninasca lorsqu'il distingue la saisie impressive, la saisie molaire et la saisie sémantique qui régissent trois formes de sémiotique ou trois types de rationalités dans lesquelles un sujet peut se trouver défini. 18. J. Geninasca parle en ces termes du «Sujet du Discours»: «Énoncer, accomplir un acte de discours, cela revient à établir comme texte et à instaurer comme discours un objet textuel en fonction d'un principe de cohérence et d'intelligibilité, mais c'est aussi assumer comme "vrai" (conforme à ce qui, conditionnant le sentiment d'identité de soi et de réalité du monde et d'autrui, fonde le sens-pourle-sujet) l'acte en quoi consiste la signification d'un énoncé discursif. Le sujet d'un acte ou (s'agissant d'une organisation discursive complexe) d'une hiérarchie d'actes discursifs se caractérise doublement, par une compétence sémiotique (une rationalité) et par une compétence et une existence modale, ou croire, si l'on convient d'appeler "croire" le monde d'inscription d'un sujet (ou d'une configuration de sujets) sur la dimension du vouloir " $(1990$ : 23-24).

19. Cet enchaînement pourrait être considéré d'un point de vue intratextuel (les figures s'enchaînent et s'interprètent dans le champ d'un texte dont le lecteur opère la mise en discours), mais aussi d'un point de vue inter-textuel (les figures s'appellent de discours en discours, tendues entre leurs reprises, leurs écarts et leur accomplissement). 20. Ces positions d'excès et de défaut ont bien été mises en lumière en sémiotique. Voir Greimas (1987); Greimas et Fontanille (1991); Zilberberg (2006). 


\section{RÉFÉREN CES BIBLIO GRAPHIQ U ES}

CALloud, J. [1993]: «Le texte à lire», dans CADIR (sous la dir. de L. Panier), Le Temps de la lecture. Exégèse biblique et sémiotique, Paris, Cerf, 31-63.

FontAnille, J. et C. Zilberberg, C. [1998] : Tension et Signification, Bruxelles, Pierre Mardaga.

GENINASCA, J. [1990]: «Du texte au discours littéraire et à son sujet », Nouveaux Actes Sémiotiques, no 10-11, 23-24.

Greimas, A.J. [1983]: Du Sens II, Paris, Seuil;

[1987]: De l'imperfection, Périgueux, Fanlac;

[1993]: «La parabole, une forme de vie», dans CADIR (sous la

dir. de L. Panier), Le Temps de la lecture. Exégèse biblique et sémiotique,

Paris, Cerf, 380-387.

GreimAS, A. J. et J. COURTÉs [1979]: Sémiotique. Dictionnaire raisonné de la théorie du langage, tome 1 , Paris, Hachette.

Greimas, A. J. et J. FonTANILLE [1991]: Sémiotique des passions. Des états de choses aux états d'âme, Paris, Seuil.

LANDOWSKI, E. [2004]: Passions sans nom, Essais de socio-sémiotique III, Paris, PUF, coll. «Formes sémiotiques".
MARTIN, F. [1995]: «Devenir des figures, ou des figures au corps», dans J. Fontanille (dir.), Le Devenir, hors-série des Nouveaux Actes Sémiotiques, Limoges, PULIM, 137-146.

PANIER, L. [1980]: «De l'idéologie dans le discours", Sémiotique et Bible, $\mathrm{n}^{\circ} 19,7-24$;

[1998]: «L'inestimable objet de l'interprétation. Approche sémiotique de la lecture », dans M. Ballabriga (dir.), Sémantique et Rhétorique, Toulouse, Éd. universitaires du Sud;

[2003a]: « Polysémie des figures et statut figural des grandeurs figuratives: l'exemple de la parabole des Mines (Évangile de Luc 19, 12-27) ", dans S. Rémi et L. Panier (dir.), La Polysémie ou l'Empire des sens. Lexique, discours, représentations, Lyon, PUL;

[2003b]: «Récit et figure dans la parabole des Mines (Luc 19). Un modèle pour une sémiotique du discours ", Modèles linguistiques, tome XXIV-1, vol. 47, 97-108;

[2008]: «Ricœur et la sémiotique: Une rencontre

“improbable"?", Semiotica, 168-1/4, 305-324.

ZILBERBERG, C. [2006] : Éléments de grammaire tensive, hors-série des Nouveaux Actes Sémiotiques, Limoges, PULIM. 